ARTICLE

Received 1 Jul 2015 | Accepted 6 Oct 2015 | Published 4 Nov 2015

DOI: 10.1038 /ncomms 9809

OPEN

\title{
Photoinduced spontaneous free-carrier generation in semiconducting single-walled carbon nanotubes
}

Jaehong Park ${ }^{1}$, Obadiah G. Reid ${ }^{1,2}$, Jeffrey L. Blackburn ${ }^{1} \&$ Garry Rumbles ${ }^{1,2,3}$

Strong quantum confinement and low dielectric screening impart single-walled carbon nanotubes with exciton-binding energies substantially exceeding $k_{\mathrm{B}} T$ at room temperature. Despite these large binding energies, reported photoluminescence quantum yields are typically low and some studies suggest that photoexcitation of carbon nanotube excitonic transitions can produce free charge carriers. Here we report the direct measurement of long-lived free-carrier generation in chirality-pure, single-walled carbon nanotubes in a low dielectric solvent. Time-resolved microwave conductivity enables contactless and quantitative measurement of the real and imaginary photoconductance of individually suspended nanotubes. The conditions of the microwave conductivity measurement allow us to avoid the complications of most previous measurements of nanotube free-carrier generation, including tube-tube/tube-electrode contact, dielectric screening by nearby excitons and many-body interactions. Even at low photon fluence (approximately 0.05 excitons per $\mu \mathrm{m}$ length of tubes), we directly observe free carriers on excitation of the first and second carbon nanotube exciton transitions.

\footnotetext{
${ }^{1}$ National Renewable Energy Laboratory, Chemistry and Nanoscience Center, 15013 Denver West Parkway, Golden, Colorado 80401, USA. ${ }^{2}$ Renewable and Sustainable Energy Institute, University of Colorado at Boulder, Boulder, Colorado 80309, USA. ${ }^{3}$ Department of Chemistry and Biochemistry, University of Colorado at Boulder, Boulder, Colorado 80309, USA. Correspondence and requests for materials should be addressed to G.R. (email: Garry.Rumbles@nrel.gov).
} 
$\mathrm{P}$ hotoinduced free-carrier generation in semiconducting single-walled carbon nanotubes (SWCNTs) has been controversial because of the substantial binding energy (hundreds of $\mathrm{meV}$ ) of photogenerated excitons, coulombically bound electron-hole pairs ${ }^{1,2}$. Although a low probability of exciton dissociation is expected in SWCNTs, photoinduced carrier generation has been observed in neat SWCNT samples in a number of studies ${ }^{3-12}$. Most of these examples of carrier generation have been observed in solid-state samples featuring either tube-tube contacts or tube-electrode contacts. As these interfaces can likely serve as carrier-generation sites, they obscure the intrinsic properties of the individual nanotubes. Examples of these potential solid-state artefacts include heterogeneous chiralities of SWCNTs that may form type-I or type-II energylevel alignments in SWCNT bundles, electrostatic screening effects in SWCNT aggregates that can enhance free-carrier generation, SWCNTs on substrates in air that often become $p$ doped or potential morphological defects or contacts with electrodes in which electric fields can dissociate excitons $3,6,7,11,13,14$. Therefore, the prevalence of intrinsic carrier generation processes in well-isolated SWCNTs remains unclear. Importantly, such unintentional carrier generation can be detrimental to applications requiring long exciton lifetimes and high luminescence quantum yields, such as biological imaging and photovoltaics ${ }^{15-17}$.

Recent solution-phase photoluminescence and transient absorption studies have suggested that charges are generated at relatively high incident photon fluences in aqueous dispersions ${ }^{9,18}$. However, it is important to note that the exciton binding energy is predicted to decrease dramatically with increasing dielectric constant of the solvent $\left(\varepsilon_{\mathrm{r}}\left(\mathrm{H}_{2} \mathrm{O}\right)=80.1\right.$, ref. 19), and high incident photon fluences can reduce the exciton binding energy via screening, facilitate exciton-exciton collisions that lead to Auger-like photoionization or even damage the CNT surface to generate defects.

In this study we demonstrate that free charge generation takes place in individual SWCNTs suspended in toluene $\left(\varepsilon_{\mathrm{r}}=2.38\right.$, ref. 19), even at ultra-low excitation fluences, which rules out both high dielectric and multi-exciton effects. We use flash-photolysis time-resolved microwave conductivity ( $f p$-TRMC) on solutionphase samples, to study the complex photoconductance of individually suspended (7,5)-chirality-enriched SWCNTs $((7,5)-S W C N T s)$. The high sensitivity of this technique allows us to use excitation fluences that are much lower than has previously been possible (lower than $10^{11}$ photons per $\mathrm{cm}^{2}$ ); the lowest excitation fluences correspond to absorbed photon densities of approximately $0.04-0.06$ photons per $\mu \mathrm{m}$ length of
$(7,5)$-SWCNTs. We find that the low-fluence yield mobility product $\Phi \sum \mu$, which is the product of charge-carrier generation efficiency $\Phi$ and the sum $\sum \mu$ of electron and hole mobilities $\mu_{\mathrm{e}}+\mu_{\mathrm{h}}$ in isolated $(7,5)$-SWCNTs is approximately 0.17 and $0.4 \mathrm{~cm}^{2} \mathrm{~V}^{-1} \mathrm{~s}^{-1}$ following $\mathrm{S}_{11}$ and $\mathrm{S}_{22}$ photoexcitation, respectively.

\section{Results}

Dispersion of $(7,5)$-SWCNTs in toluene. The electronic absorption spectrum of $(7,5)-S W C N T s$ suspended in toluene by poly[9,9-dioctylfluorenyl-2,7-diyl] (PFO) is shown in Figure 1a. Employing established methods with PFO as a dispersing agent ${ }^{20,21}$, we obtain highly $(7,5)$-chirality enriched SWCNT solution and remove excess PFO until a polymer:SWCNT mass ratio of approximately 4 is obtained (see Methods for full details of sample preparation). The distinctive $\mathrm{S}_{11}$ and $\mathrm{S}_{22}$ excitonic transitions of $(7,5)$-SWCNTs, peaking at 1,044 and $653 \mathrm{~nm}$, respectively, confirm the purity of the $(7,5)$ SWCNT solution sample. In addition, electronic absorption data preclude the possibility that PFO is excited in the range from 450 to $1,200 \mathrm{~nm}$. The energy-level diagram shown in Figure $1 \mathrm{~b}$ illustrates that the PFO polymers ${ }^{22,23}$ and the $(7,5)-$ SWCNTs $^{24}$ form a type-I heterostructure and, therefore, it is evident that the $(7,5)-S_{W C N T}{ }^{*}$ state generated via $S_{11}$ excitation should not exhibit electron/energy transfer from $(7,5)$-SWCNTs to PFO polymers, which is consistent with previous literature results ${ }^{25}$.

Probing photoinduced free-carrier generation of SWCNTs. We employ a cavity-based $f p$-TRMC technique to explore photoinduced free-carrier generation and recombination dynamics in the solution-phase $(7,5)-S W C N T s$. The use of the resonance cavity in $f p$-TRMC experiments allows us to operate at very low excitation photon fluences (approximately $10^{10}-10^{12}$ photons per $\mathrm{cm}^{2}$ ) and to explicitly measure the complex conductivity, avoiding complications from many-body interactions such as excitonexciton annihilation processes. In this regard, $f p$-TRMC has shown its versatility in determining the charge-carrier mobility, charge-carrier generation efficiency and subsequent carrier recombination dynamics for various conjugated polymer aggregates in solution, and thin-film layers, including organic layers or hybrid organic-inorganic layers ${ }^{7,26-38}$. Figure 2 a displays representative time-resolved TRMC transients for $(7,5)-S W C N T s$ suspended in toluene, as well as those for controls of PFO dissolved in toluene and neat toluene solvent in Supplementary Figure 1; the vertical axis is the change in microwave power reflected from the cavity at the resonance frequency. No reflected microwave transient
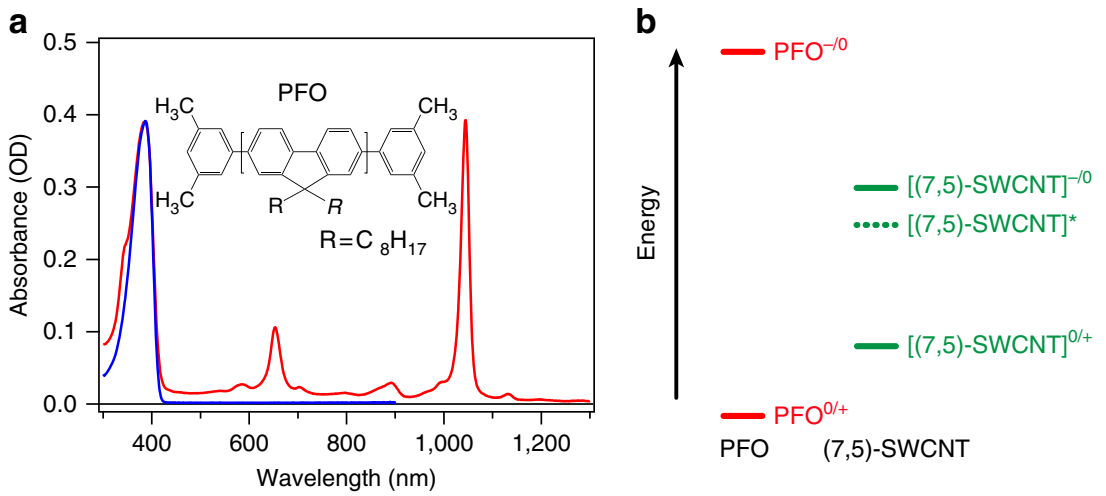

Figure 1 | (7,5)-SWCNT dispersion wrapped with PFO polymer. (a) Electronic absorption spectra of (7,5)-SWCNTs suspended in toluene via polymer wrapping in PFO (in red) and of PFO polymer dissolved in toluene (in blue), respectively (see Methods for sample preparation). (b) Energy-level diagram illustrating that the PFO polymer and (7,5)-SWCNTs form a type-I heterostructure. A dotted line depicts the $\mathrm{S}_{11}$ state of $[(7,5)-\text { SWCNT }]^{*}$. 


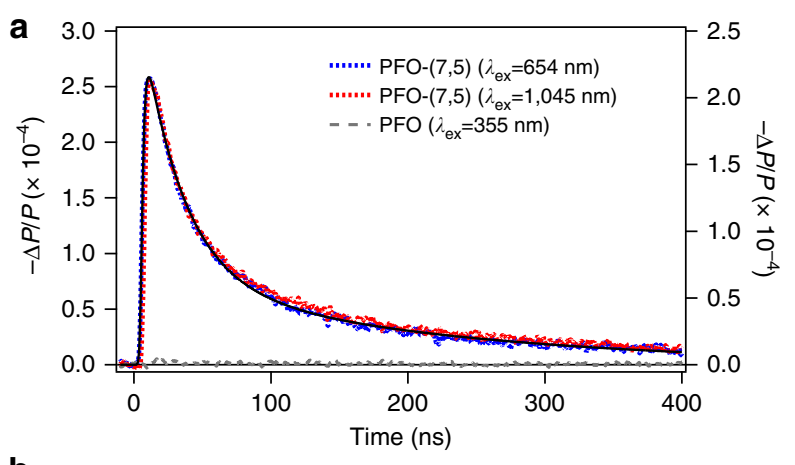

b

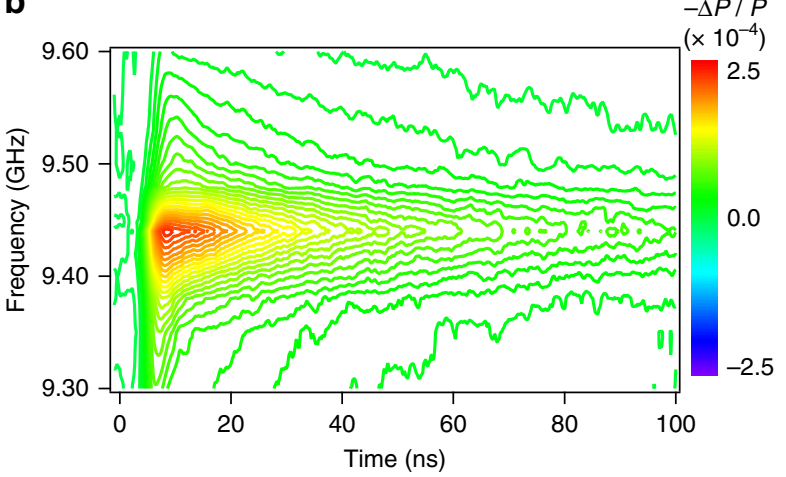

Figure 2 | Phtoinduced free-carrier generation probed by microwave conductivity. (a) Representative time-resolved reflected microwave transients for (red and blue) (7,5)-SWCNTs suspended in toluene and (grey, left $y$ axis) PFO dissolved in toluene. The SWCNT transients result from photoexcitation at either the $S_{22}$ transition (blue, left $y$ axis) or $S_{11}$ transition (red, right $y$ axis). (b) Frequency-dependent reflected microwave power transients for $(7,5)$-SWCNTs suspended in toluene, following excitation at $\mathrm{S}_{22}$. In $\mathbf{a}$, a biexponential fit is displayed as the solid black line. Experimental conditions: the excitation photon fluence was approximately 1.0-1.5 $\times 10^{12}$ photons per $\mathrm{cm}^{2}$ for the data presented in a and $4.4 \times 10^{12}$ photons per $\mathrm{cm}^{2}$ for $\mathbf{b}$; $5 \mathrm{~ns}$ pulse width; room temperature.

signals are observed with either neat PFO dissolved in toluene (grey dashed line in Figure 2a, left $y$ axis) or solvent alone (Supplementary Figure 1), showing that the change of microwave absorption is due solely to $(7,5)$-SWCNTs. $f p$-TRMC experiments for a variety of (7,5)-SWCNT concentrations (1.23-12.3 nM per $\mu \mathrm{m}$ length of $(7,5)$-SWCNTs) reveal that the transient decay dynamics are insensitive to concentration within this range (Supplementary Figure 2).

Frequency-resolved microwave conductivity measurements. The details of the solution-phase $f p$-TRMC experimental setup and discussion are described in Supplementary Figures 3-6 and Supplementary Methods, and the theoretical background of $f p$-TRMC can be found elsewhere ${ }^{28,29,39,40}$. In brief, in the most general terms, $f p$-TRMC experiments measure the time evolution of the complex dielectric constant $\varepsilon$ of the sample after photoexcitation. The present experiments are conducted with the sample mounted in a microwave cavity and the complex dielectric constant of the sample is calculated from the cavity resonance characteristics. Changes in the real part of the dielectric constant lead to a shift in the resonance frequency, whereas the imaginary part determines microwave power loss in the cavity. Charges photogenerated in the sample (photoconductivity) can contribute to both the real and the imaginary parts of the dielectric constant depending on their mobility and degree of confinement ${ }^{40}$. Conductivity can be expressed in terms of dielectric constant as:

$$
\sigma=i \omega \varepsilon=\varepsilon_{0} \omega\left(i \varepsilon^{\prime}+\varepsilon^{\prime \prime}\right)
$$

where $\sigma, \omega, \varepsilon_{0}, \varepsilon^{\prime}$ and $\varepsilon^{\prime \prime}$ represent the complex conductivity, the radian frequency of the microwave electric field, the vacuum permittivity, and the real and imaginary parts of the dielectric constant at frequency $\omega$, respectively. Thus, the real part of the conductivity is identified with the imaginary part of the dielectric constant-microwave absorption. An important caveat here is that real conductivity is only one of two possible components of the loss term. Dielectric loss can also contribute, as when molecular dipoles re-orient in the field. These two loss mechanisms are indistinguishable in the TRMC experiment and we rely on arguments later in the text, to show that a significant dielectric loss contribution to the signals we observe is unlikely.

$f p$-TRMC measurements made only at a single resonance frequency cannot distinguish between the real and imaginary parts of the conductivity, because a frequency shift simply modulates the reflected power, much as a change in power absorption would ${ }^{41}$. Frequency-dependent measurements are therefore necessary to identify the origin of the transient signals in $(7,5)$-SWCNTs. A set of reflected power transients were collected at 13 different microwave frequencies, spanning the cavity resonance curve, following $S_{22}$ excitation of a $(7,5)$-SWCNT solution. Figure $2 \mathrm{~b}$ and Supplementary Figure 7 show contour plots of frequency-dependent reflected microwave power transients generated from these data and exhibit a negligible frequency shift as a function of time, implying that the transient signals obtained on resonance are dominated by a change in the real conductivity of the sample. These results conclusively demonstrate that photoexcitation of $(7,5)$-SWCNTs generates some amount of mobile free carriers.

Transient photoconductance decay. Under both $S_{11}$ and $S_{22}$ excitation conditions, microwave transient decay dynamics normalized at the peak are indistinguishable as shown in Figure 2a. In a previous study of SWCNT thin films, the photoconductance $\Delta G$ signal, which is proportional to the reflected microwave power $-\Delta P / P$, decayed by approximately $90 \%$ within the first $10 \mathrm{~ns}$ (ref. 7). In our current solution-phase fp-TRMC measurements, the transient signals persist much longer. Both transient decay profiles are fitted using a biexponential function with time constants $\tau_{\mathrm{i}}$ and associated amplitudes $a_{\mathrm{i}}$ of $\tau_{1}=27 \mathrm{~ns}$ $\left(a_{1}=0.75\right)$ and $\tau_{2}=212 \mathrm{~ns}\left(a_{2}=0.25\right)$, and yield the average lifetime $\tau_{\text {avg }}$ of $161 \mathrm{~ns}$, from $\tau_{\text {avg }}=\Sigma_{\mathrm{i}} f_{\mathrm{i}} \tau_{\mathrm{i}}$, where $f_{\mathrm{i}}$ is the fractional contribution of each time constant, which is $\left(a_{\mathrm{i}} \tau_{\mathrm{i}}\right) / \Sigma_{\mathrm{j}} a_{\mathrm{j}} \tau_{\mathrm{j}}$. The different transient decay behaviour between solution-phase individualized SWCNTs and thin-film SWCNTs suggest that inter-tube junctions in SWCNT thin films possibly facilitate carrier recombination by serving as recombination sites $^{42}$. In contrast, the longer-lived solution-phase TRMC transient decay dynamics probably represent more intrinsic intra-tube carrierrecombination dynamics, as inter-tube contact is prohibited in the highly individualized SWCNTs.

Photoconductance action spectrum. Figure 3a displays the action spectrum of the peak reflected microwave power for $(7,5)$-SWCNTs suspended in toluene, which examines the correlation of carrier generation with excitation wavelength. Photoconductance values in the action spectrum are taken at low photon fluence $\left(I_{0}<5 \times 10^{12}\right.$ photons per $\left.\mathrm{cm}^{2}\right)$ and are normalized for excitation photon fluence. The shape of the action spectrum closely matches the absorptance spectrum of $(7,5)$-SWCNTs, demonstrating further that the origin of the photoconductance is indeed (7,5)-SWCNTs. Equations (2) and (3) show the 

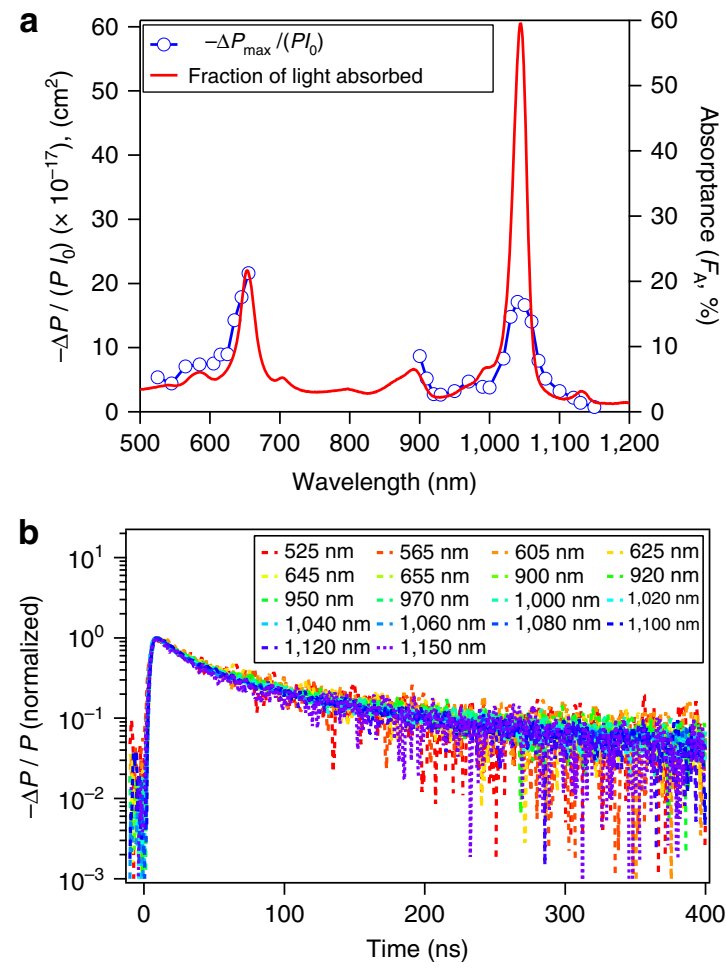

Figure 3 | Photoconductance action spectrum and excitation wavelength dependence of photoconductance transients. (a) Action spectrum for peak reflected microwave signal $(\Delta P / P$, end of pulse) normalized by the excitation photon fluence $\left(I_{0}\right)$ for $(7,5)$-SWCNTs suspended in toluene (blue, left $y$ axis). Electronic absorptance spectrum of (7,5)-SWCNTs suspended in toluene is overlaid for comparison (red, right $y$ axis). (b) Normalized reflected microwave transients decay for a variety of excitation wavelengths noted in the figure. Experimental conditions: $I_{0}<5 \times 10^{12}$ photons per $\mathrm{cm}^{2}$; room temperature.

relation between the reflected microwave power and the carriergeneration yield.

$$
\begin{gathered}
-\frac{\Delta P_{\max }}{P}=K \Delta G_{\max }=K \beta q_{\mathrm{e}}\left(\Phi \sum \mu\right)\left(I_{0} F_{\mathrm{A}}\right) \\
-\frac{\Delta P_{\max }}{\left(P \times I_{0}\right)}=1.381 \times 10^{-15} \times \sum \mu\left(\Phi F_{\mathrm{A}}\right)\left[\mathrm{cm}^{2}\right]
\end{gathered}
$$

In equations (2) and (3), $I_{0}$ (photons per $\mathrm{cm}^{2}$ per pulse) is the excitation photon fluence, $F_{\mathrm{A}}$ is the fraction of light absorbed at the excitation wavelength (absorptance), $K(\Omega)$ is a sensitivity factor, determined as 2,400 from the cavity resonance characteristics and the dielectric properties of the medium (see Supplementary Methods for evaluation of $K$ factor), $\beta$ is the ratio between the long and short axes of the sample cross-section that is perpendicular to the microwave propagation vector, $q_{\mathrm{e}}(\mathrm{C})$ is the elementary charge and $\sum \mu$ is the sum of electron and hole mobilities. The reflected microwave power normalized by the incident photon fluence $-\Delta P /\left(P I_{0}\right)$ is proportional to $\Phi F_{\mathrm{A}}$, as the mobilities can be assumed constant. Therefore, by comparing $-\Delta P /\left(P I_{0}\right)$ with the absorptance spectrum, we can extract the relative carrier-generation yield for $S_{11}$ and $S_{22}$ excitation. Interestingly, the action spectrum comparing $\Phi F_{\mathrm{A}}$ near $\mathrm{S}_{11}$ and $\mathrm{S}_{22}$ transitions (Figure 3a) shows that the carrier-generation quantum yield $\Phi$ under $S_{22}$ excitation is about three times higher than that under $S_{11}$ excitation.

Recently, Kumamoto et al. ${ }^{11}$ reported photocurrent with $\mathrm{S}_{22}$ excitation for an individual $(10,6)$-SWCNT grown on a Si substrate. Although the excitation wavelengths of their experiments reside within the $S_{22}$ spectral domain, they clearly identified the presence of photocurrent with $S_{22}$ excitation and non-zero conductivity even with zero applied bias, suggesting that the $S_{22}$ exciton dissociation is a spontaneous process. In addition, Kazaoui et al. ${ }^{10}$ observed qualitatively higher photocurrent quantum yield with $S_{22}$ excitation over that with $S_{11}$ excitation for a $(7,5)$-SWCNT thin film. Likewise, as we probe photoinduced carrier generation in a low dielectric solvent, our results also suggest that exciton dissociation in $(7,5)$ SWCNTs is unlikely due to an electric field and more likely a spontaneous process in SWCNTs.

To rule out the possibility that the PFO wrapping the nanotube provides a locally higher dielectric constant, we have performed microwave cavity resonance measurements as a function of PFO:toluene mass ratio and compared them with analogous experiments where a higher dielectric constant solvent is added to the toluene. If the guest molecule added to neat toluene possesses a different dielectric constant from toluene, then the resonance frequency of the loaded microwave cavity will shift in response. The fact that the cavity resonance position does not shift detectably as PFO is added, in contrast to many of the more polar guest solvents, suggests that the PFO polymer has essentially the same dielectric constant as the toluene and does not provide a high local dielectric environment around the nanotubes (see Supplementary Figure 8 and Supplementary Methods for microwave cavity resonance measurements as a function of a guest molecule:toluene mass ratio).

As the action spectrum of the peak reflected microwave power (normalized for excitation photon fluence) near the $S_{22}$ transition of $(7,5)$-SWCNTs appears to match its excitonic features, no other intermediate state seems to be involved between photoexcitation and $\mathrm{S}_{22}$ exciton formation. This correlation suggests that carriers are produced from $S_{22}$ excitons and carrier generation seems to compete with the $S_{22} \rightarrow S_{11}$ internal conversion process that is known to be very fast (faster than $50 \mathrm{fs})^{43}$. Given the exciton binding energy (approximately $0.4 \mathrm{eV}$ ) for $(7,5)$-SWCNTs ${ }^{1,2}$, the continuum states for the $S_{11}$ exciton lie below the lowest unoccupied molecular orbital of the $S_{22}$ state. Autoionization of $S_{22}$ excitons via populating a vibronically hot $S_{11}$ state or free-carrier continuum states has been proposed for the charge-generation mechanism previously ${ }^{5,44}$, and it should be noted that in our action spectrum the carrier-generation yield with excitation at the $S_{11}$ phonon side band (approximately $900 \mathrm{~nm}$ ) appears to be comparable to that of $S_{22}$ excitation. Figure $3 \mathrm{~b}$ demonstrates identical microwave-transient decay profiles for widely varying excitation wavelengths from $S_{11}$ to energies higher than $S_{22}$. The similarity of these transients suggests that the initial photoproducts from either $S_{11}$ or $S_{22}$ excitation do not have an impact on the carrier decay dynamics, implying that the mobile carriers generated from photoexcitation are the same species, regardless of excitation energy. It should be noted that, although these $<1-\mathrm{nm}$ diameter SWCNTs should have $S_{11}$ exciton binding energies of approximately $0.4 \mathrm{eV}^{1,2}$, carrier generation following $S_{11}$ excitation even at lower excitation fluences (lower than $10^{11}$ photons per $\mathrm{cm}^{2}$; Figure $4 \mathrm{a}$ ) is unambiguously observed, although the yield is one-third of that observed for $\mathrm{S}_{22}$ excitation.

Excitation fluence-dependent yield-mobility product. The figure of merit extracted from $f p$-TRMC using equations (2) is the product $\Phi \sum \mu$ of the free-carrier yield $\Phi$ and the sum of mobilities $\sum \mu$, which is proportional to the photoconductance $\Delta G$ normalized by the absorbed photon fluence $I_{0} F_{\mathrm{A}}$. Figure $4 \mathrm{a}, \mathrm{b}$ (and further in Supplementary Figure 9) displays the time-resolved $f p$-TRMC results of toluene-suspended $(7,5)$-SWCNTs following $\mathrm{S}_{11}$ and $\mathrm{S}_{22}$ excitations, respectively, where the $y$ axis has been 

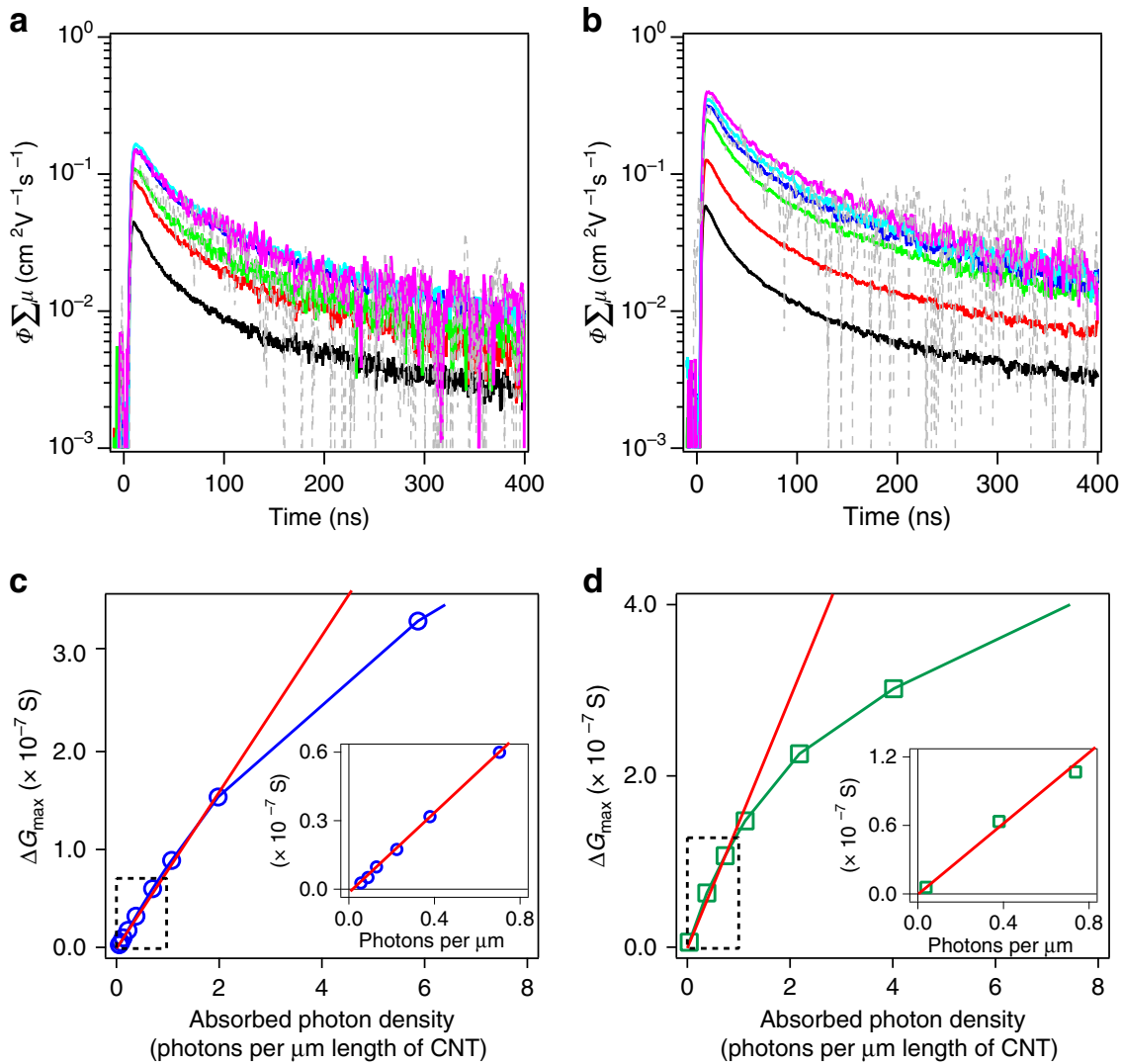

e

Absorbed photon density (photons per $\mu \mathrm{m}$ of CNT)

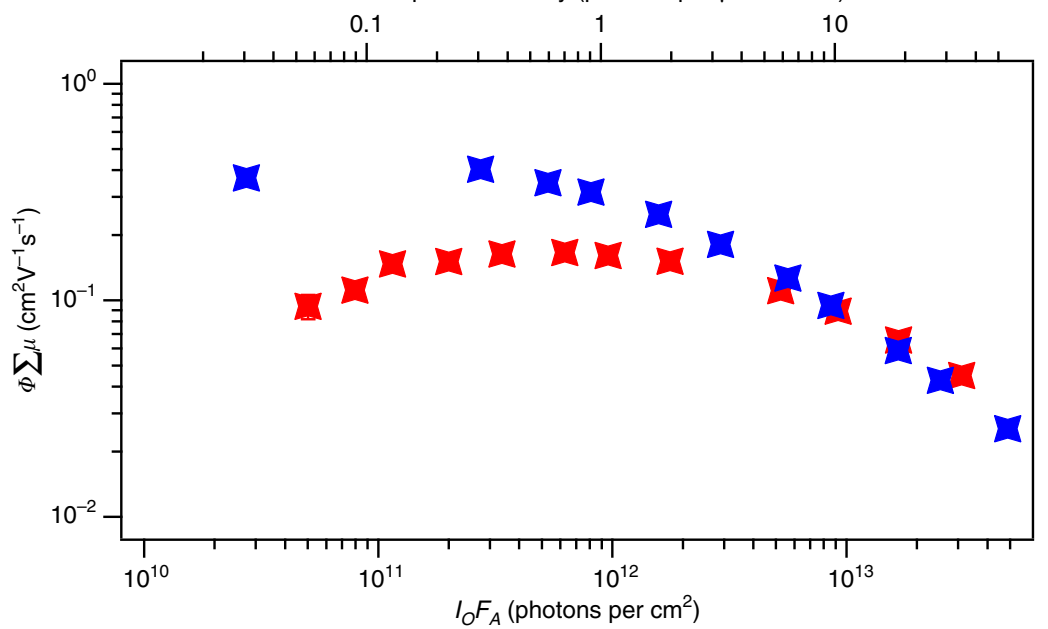

Figure 4 | Excitation fluence-dependent photoconductance. $(\mathbf{a}, \mathbf{b})$ The dependence of the yield mobility product $\Phi \sum \mu$ transient decays on excitation photon fluence $\left(I_{0}\right)$ for excitation of the $(\mathbf{a}) S_{11}$ transition (black, red, green, blue, cyan, purple and grey: 3,470 $\times 10^{10}, 1,030 \times 10^{10}, 584 \times 10^{10}, 197 \times 10^{10}$, $70.0 \times 10^{10}, 22.3 \times 10^{10}$ and $8.87 \times 10^{10}$ photons per $\mathrm{cm}^{2}$, respectively) and (b) $\mathrm{S}_{22}$ transition (black, red, green, blue, cyan, purple and grey: $4,520 \times 10^{10}$, $1,520 \times 10^{10}, 427 \times 10^{10}, 220 \times 10^{10}, 144 \times 10^{10}, 74.1 \times 10^{10}$ and $7.41 \times 10^{10}$ photons per $\mathrm{cm}^{2}$, respectively). (c, d) The peak of reflected microwave transients, $\Delta G_{\max }$ (end of pulse), evincing the linearity of peak reflected transient signals below the absorbed photon density of approximately 0.8 photon per $\mu \mathrm{m}$ length of $(7,5)$-SWCNTs for both (c) $S_{11}$ and (d) $S_{22}$ transitions. The red solid lines represent a linear function. (e) $\Phi \sum \mu$ as a function of absorbed photon fluence for $(7,5)$-SWCNTs suspended in toluene with exciting at (blue) $S_{22}$ or (red) $S_{11}$ transitions, respectively. The top $x$ axis corresponds to the absorbed photons per $\mu \mathrm{m}$ length of $(7,5)$-SWCNTs for given $I_{0} F_{\mathrm{A}}$ (bottom $x$ axis).

converted to $\Phi \sum \mu$. On photoexcitation at the $\mathrm{S}_{11}$ transition, $\Phi \sum \mu$ values can be as high as approximately $0.17 \mathrm{~cm}^{2} \mathrm{~V}^{-1} \mathrm{~s}^{-1}$ in a variety of excitation photon fluences $I_{0}$ ranging from $5.6 \times 10^{10}$ to approximately $3.5 \times 10^{13}$ photons per $\mathrm{cm}^{2}$, corresponding to an absorbed photon density of approximately $0.056-$ 35 photons per $\mu \mathrm{m}$ length of $(7,5)$-SWCNTs $\left(\Phi \sum \mu\right.$ transient data from $5.6 \times 10^{10}$ photons per $\mathrm{cm}^{2}$ is provided in Supplementary
Figure 9, see Supplementary Methods for the calculation of absorbed photon density in (7,5)-SWCNTs). Assuming that one absorbed photon creates one exciton, the absorbed photon density per $\mu \mathrm{m}$ length of $(7,5)$-SWCNTs corresponds to the initial exciton population per $\mu \mathrm{m}$ length of $(7,5)$-SWCNTs (hereafter abbreviated as $\left.[\mathrm{Ex}]_{\mu \mathrm{m}}\right)$. On the other hand, $\Phi \sum \mu$ values following excitation of the $S_{22}$ transition are as high as approximately 
$0.4 \mathrm{~cm}^{2} \mathrm{~V}^{-1} \mathrm{~s}^{-1}$ with excitation fluences below $7.4 \times 10^{11}$ photons per $\mathrm{cm}^{2}$, which is about 2.4 times higher than the maximum $\Phi \sum \mu$ observed for $\mathrm{S}_{11}$ excitation. As the carrier mobility can be assumed to be constant between $S_{11}$ and $S_{22}$ excitation, the 2.4 times higher $\Phi \sum \mu$ on photoexcitation of the $S_{22}$ transition suggests the carrier-generation yield with $S_{22}$ excitation is approximately 2.4 times higher than that with $S_{11}$ excitation. This result is consistent with the $f p$-TRMC action spectrum results in Figure 3a.

Even with approximately three orders of magnitude fluence increase, the microwave transient decay dynamics appear to be insensitive to the exciton density, as shown in the normalized transient decay profiles in Supplementary Figure 9. This result infers that in this exciton density range $\left(0.056-35[\mathrm{Ex}]_{\mu \mathrm{m}}\right.$ of $(7,5)-S W C N T s)$, carrier recombination effectively occurs through a first-order process and the interactions between a carrier and a neighbouring carrier created from another exciton are negligible for carrier recombination. The absence of fluence dependence within our excitation conditions $\left(I_{0}\right.$ between approximately $8 \times 10^{10}$ and $4000 \times 10^{10}$ photons per $\mathrm{cm}^{2}$ ) under our very low exciton density presumably infers a geminate carrier recombination process. Considering that our TRMC technique is detecting only free carriers, this carrier recombination is best described as a secondary geminate recombination process. Secondary geminate recombination requires dissociation and separation of the charges initially bound as an exciton, whereby subsequent recombination is diffusion mediated. The fairly long average transient decay lifetime (approximately $160 \mathrm{~ns}$ ) suggests that one carrier might be trapped and only the other carrier is mobile.

Although we cannot attribute previously observed all longlived (above the ns time domain) excited population in solutionphase studies to free carriers ${ }^{45,46}$, using those yields (approximately 3-10\%) we can estimate the lower limit of freecarrier mobilities. At a conservative estimate for free-carrier mobilities with $10 \%$ yield, assuming only one carrier species is mobile, $9 \mathrm{GHz}$ free-carrier mobility of $(7,5)-S W C N T s$ is calculated to be higher than $4 \mathrm{~cm}^{2} \mathrm{~V}^{-1} \mathrm{~s}^{-1}$ with $\mathrm{S}_{22}$ excitation. This estimated free-carrier mobility is comparable to the previous literature values of semiconducting SWCNT thin-film carrier mobilities that range from 1.3 to $2 \mathrm{~cm}^{2} \mathrm{~V}^{-1} \mathrm{~s}^{-1}$ (refs 7, 47). On the other hand, when using higher available literature values of SWCNT carrier mobility ${ }^{48}$, we can instead speculate the lower limit of the carrier-generation yield, which is $0.04 \%$ using approximately $1,000 \mathrm{~cm}^{2} \mathrm{~V}^{-1} \mathrm{~s}^{-1}$.

Figure $4 c, d$ show that the maximum photoconductance $\Delta G_{\max }$ extracted from the photoconductance peak intensity increases linearly with exciton density below approximately $0.8[\mathrm{Ex}]_{\mu \mathrm{m}}$ of $(7,5)$-SWCNTs. These results imply that carrier generation in this low fluence regime (lower than $0.8[\mathrm{Ex}]_{\mu \mathrm{m}}$ ) is not a bimolecular process, although we cannot rule out the possibility that at a higher excitation intensity exciton-exciton interactions could mediate exciton dissociation ${ }^{7,9}$.

Figure $4 \mathrm{e}$ compares $\Phi \sum \mu$ as a function of $I_{0} F_{\mathrm{A}}$ with either $\mathrm{S}_{11}$ (red) or $\mathrm{S}_{22}$ (blue) excitation. At low $I_{0} F_{\mathrm{A}}$, where many-body interactions such as exciton-exciton annihilation or excitoncarrier annihilation are negligible, and assuming carrier mobility is constant, then $\Phi \sum \mu$ should be independent of $I_{0} F_{\mathrm{A}}$. As a result, $\Phi \sum \mu$ exhibits a plateau at sufficiently low $I_{0} F_{\mathrm{A}}$ range, which has been observed in many other neat thin films or donor-acceptor systems ${ }^{29,30,34,49}$. This plateau suggests that photoinduced carrier generation is a pseudo first-order reaction at low fluence. Likewise, $\Phi \sum \mu$ exhibits a plateau at exciton density below approximately $0.8-1[\mathrm{Ex}]_{\mu \mathrm{m}}$ and begins to decrease with $I_{0} F_{\mathrm{A}}$ increase, as a result of many-body interactions ${ }^{7}$. It is important to note that transient signals are present even at approximately $0.06[\mathrm{Ex}]_{\mu \mathrm{m}}$ of $(7,5)$ SWCNTs with the $S_{11}$ excitation (much less than one exciton per
SWCNT on average), suggesting that photoinduced carrier generation at these low fluences does not result from excitonexciton interactions. Given the previous study showing electricfield dependence of photocurrent generation ${ }^{6}$, we conjecture that local electric fields present at tube ends or defects could induce exciton dissociation for the case of $\mathrm{S}_{11}$ excitation.

\section{Discussion}

In conclusion, we use a solution-phase $f p$-TRMC measurement and individualized highly (7,5)-chirality-enriched SWCNT samples dispersed in toluene by the PFO. We probe photoinduced mobile carrier generation in highly isolated SWCNTs in a low dielectric solvent (toluene, $\varepsilon_{\mathrm{r}}=2.38$ ) at very low exciton densities (lower than 0.06 excitons per $\mu \mathrm{m})$. Even under these mild conditions, we unambiguously observe photoconductance in well-isolated $(7,5)$ SWCNTs with a yield-mobility product of approximately 0.17 and $0.4 \mathrm{~cm}^{2} \mathrm{~V}^{-1} \mathrm{~s}^{-1}$ following $\mathrm{S}_{11}$ and $\mathrm{S}_{22}$ photoexcitation, respectively. The carrier-generation quantum yield with $S_{22}$ excitation appears approximately 2.4 times higher than that with $S_{11}$ excitation, suggesting that the autoionization of $S_{22}$ excitons enhances the carrier-generation efficiency. In contrast, the transient decay dynamics are independent of excitation wavelength, suggesting that the mobile carriers generated from either $S_{11}$ or $S_{22}$ excitation undergo identical decay pathways, that is, trapping, recombination, etc. This study demonstrates that frequencyresolved solution-phase $f p$-TRMC is beneficial to interrogate carrier dynamics of SWCNTs, as selective monitoring of free carriers is achievable with relatively fast ns-time resolution, and photoinduced processes can be studied at extremely low excitation fluence conditions (lower than $10^{11}$ photons per $\mathrm{cm}^{2}$ ). In addition, in solution-phase $f p$-TRMC, morphology-mediated information can be avoided, to investigate intrinsic material properties and dynamics. With these advantages in mind, this frequency-resolved solution-phase $f p$-TRMC technique can be applied to a variety of other nanomaterial systems.

\section{Methods}

PFO-(7,5) SWCNTs purification and sample preparation. SWCNT powder was added to approximately $2 \mathrm{mg} \mathrm{ml}^{-1} \mathrm{PFO}$ solution in toluene such that the final weight ratio between SWCNT powder and PFO in toluene becomes 1:2 and the solution was dispersed through tip sonication ( $1 / 2$ in probe) for $30 \mathrm{~min}$ at $40 \%$ intensity (Cole-Palmer CPX 750$)$ in a bath of cool $\left(18^{\circ} \mathrm{C}\right)$ flowing water. The dispersion was then centrifuged using an SW32Ti rotor (Beckman) at 13,200 r.p.m. and $20^{\circ} \mathrm{C}$ for $10 \mathrm{~min}$. The supernatant, containing highly $(7,5)$-enriched SWCNTs, was then collected. The (7,5)-SWCNT dispersion was then centrifuged at 24,100 r.p.m. and $0{ }^{\circ} \mathrm{C}$ for $20 \mathrm{~h}$, to remove excess solution-phase (unbound) PFO and to concentrate the $(7,5)$-SWCNTs for the $f p$-TRMC experiments. In this case, the resulting supernatant (containing free solution-phase PFO polymer) was discarded and the pellet (containing the $(7,5)$-SWCNT material) was redispersed in toluene. After this process, the PFO:SWCNT mass ratio is approximately $4: 1$. To calculate PFO:SWCNT mass ratio, the PFO mass extinction coefficient was experimentally determined in toluene as $95.7 \mathrm{lg}^{-1} \mathrm{~cm}^{-1}\left(\varepsilon_{387 \mathrm{~nm}}=37,000 \mathrm{M}^{-1} \mathrm{~cm}^{-1}\right.$ for fluorene repeating unit in toluene).

fp-TRMC experiments. The details of $f p$-TRMC experimental setup and its theoretical background have been reported elsewhere $28,29,39,40$ and the full accounts of solution-phase $f p$-TRMC are provided in Supplementary Methods. A schematic instrumental layout is described in Supplementary Figure 3. Although the details of $f p$-TRMC apparatus have been reported previously ${ }^{50,51}$, several modifications to load a solution cell are necessary to carry out solution-phase $f p$-TRMC. The SWCNT solution sample is loaded in a custom-designed cuvette (5-mm beam path length), which is shown in Supplementary Figure 4. This cuvette is mounted in a pocket made of PTFE (poly tetra fluoro ethylene; Teflon) and the pocket is positioned at the brass base as shown in Supplementary Figure 4. For illuminating the cuvette, 13 holes are made in the waveguide with a diameter of $3.175 \mathrm{~mm}$, to prevent leakage of microwaves. The details of the microwave resonance cavity characterization such as $\mathrm{K}$-factor and resonance curve measurements are described in Supplementary Methods. The sample is optically excited through the pattern of 13 holes by an approximately 5 -ns full width at half maximum laser pulse from an optical parametric oscillator (Continuum Panther), pumped by the $355 \mathrm{~nm}$ harmonic of an Nd:YAG laser (Continuum Powerlite) and sample photoconductance is measured by 
monitoring the transient change in microwave power absorption by the sample after the laser pulse. The excitation power is adjusted with a series of neutral density filters. To measure an incident excitation power through the 13-hole pattern, we manufacture a mask with a same pattern and mount on the laser power meter sensor.

\section{References}

1. Wang, F., Dukovic, G., Brus, L. E. \& Heinz, T. F. The optical resonances in carbon nanotubes arise from excitons. Science 308, 838-841 (2005).

2. Scholes, G. D. \& Rumbles, G. Excitons in nanoscale systems. Nat. Mater. 5, 683-696 (2006).

3. Gadermaier, C. et al. Long-lived charged states in single-walled carbon nanotubes. Nano Lett. 6, 301-305 (2006).

4. Beard, M. C., Blackburn, J. L. \& Heben, M. J. Photogenerated free carrier dynamics in metal and semiconductor single-walled carbon nanotube films. Nano Lett. 8, 4238-4242 (2008).

5. Crochet, J. J. et al. Free-carrier generation in aggregates of single-wall carbon nanotubes by photoexcitation in the ultraviolet regime. Phys. Rev. Lett. 107, 257402 (2011)

6. Barkelid, M. \& Zwiller, V. Photocurrent generation in semiconducting and metallic carbon nanotubes. Nat. Photon. 8, 47-51 (2013).

7. Bindl, D. J. et al. Free carrier generation and recombination in polymerwrapped semiconducting carbon nanotube films and heterojunctions. J. Phys. Chem. Lett. 4, 3550-3559 (2013).

8. Soavi, G. et al. Ultrafast charge photogeneration in semiconducting carbon nanotubes. J. Phys. Chem. C 117, 10849-10855 (2013).

9. Yuma, B. et al. Biexciton, single carrier, and trion generation dynamics in single-walled carbon nanotubes. Phys. Rev. B 87, 205412 (2013).

10. Kazaoui, S. et al. Photocurrent quantum yield of semiconducting carbon nanotubes: dependence on excitation energy and exciton binding energy. $J$ Phys. Chem. C 118, 18059-18063 (2014).

11. Kumamoto, Y. et al. Spontaneous exciton dissociation in carbon nanotubes. Phys. Rev. Lett. 112, 117401 (2014).

12. Engel, M. et al. Photocurrent spectroscopy of (n, m) sorted solution-processed single-walled carbon nanotubes. ACS Nano 8, 9324-9331 (2014).

13. Lüer, L., Crochet, J., Hertel, T., Cerullo, G. \& Lanzani, G. Ultrafast excitation energy transfer in small semiconducting carbon nanotube aggregates. ACS Nano 4, 4265-4273 (2010).

14. Minot, E. D., Yaish, Y., Sazonova, V. \& McEuen, P. L. Determination of electron orbital magnetic moments in carbon nanotubes. Nature 428, 536-539 (2004).

15. Liu, Z., Tabakman, S., Welsher, K. \& Dai, H. Carbon nanotubes in biology and medicine: In vitro and in vivo detection, imaging and drug delivery. Nano Res. 2, 85-120 (2009).

16. D'Souza, F. \& Ito, O. Photosensitized electron transfer processes of nanocarbons applicable to solar cells. Chem. Soc. Rev. 41, 86-96 (2012).

17. Ferguson, A. J., Blackburn, J. L. \& Kopidakis, N. Fullerenes and carbon nanotubes as acceptor materials in organic photovoltaics. Mater. Lett. 90, 115-125 (2013)

18. Santos, S. M. et al. All-optical trion generation in single-walled carbon nanotubes. Phys. Rev. Lett. 107, 187401 (2011).

19. CRC Handbook of Chemistry and Physics 95th Edition (CRC Press, 2014).

20. Nish, A., Hwang, J.-Y., Doig, J. \& Nicholas, R. J. Highly selective dispersion of single-walled carbon nanotubes using aromatic polymers. Nat. Nanotechnol. 2, 640-646 (2007)

21. Guillot, S. L. et al. Precision printing and optical modeling of ultrathin SWCNT/C 60 heterojunction solar cells. Nanoscale 7, 6556-6566 (2015).

22. Fukuda, M., Sawada, K., Morita, S. \& Yoshino, K. Novel characteristics of conducting poly (9-alkylfluorene), poly (9, 9-dialkylfluorene) and poly (1, 10bis $\left(9^{\prime}\right.$-alkylfluorenyl) alkane). Synth. Met. 41, 855-858 (1991).

23. Janietz, S. et al. Electrochemical determination of the ionization potential and electron affinity of poly(9,9-dioctylfluorene). Appl. Phys. Lett. 73, 2453-2455 (1998).

24. Paolucci, D. et al. Singling out the electrochemistry of individual single-walled carbon nanotubes in solution. J. Am. Chem. Soc. 130, 7393-7399 (2008)

25. Švrček, V., Cook, S., Kazaoui, S. \& Kondo, M. Silicon nanocrystals and semiconducting single-walled carbon nanotubes applied to photovoltaic cells. J. Phys. Chem. Lett. 2, 1646-1650 (2011).

26. Gelinck, G. H., Warman, J. M. \& Staring, E. G. J. Polaron pair formation, migration, and decay on photoexcited poly (phenylenevinylene) chains. J. Phys. Chem. 100, 5485-5491 (1996).

27. Hoofman, R. J. O. M. et al. Influence of backbone conformation on the photoconductivity of polydiacetylene chains. Macromolecules 33, 9289-9297 (2000).

28. Saeki, A., Koizumi, Y., Aida, T. \& Seki, S. Comprehensive approach to intrinsic charge carrier mobility in conjugated organic molecules, macromolecules, and supramolecular architectures. Acc. Chem. Res. 45, 1193-1202 (2012).

29. Savenije, T. J., Ferguson, A. J., Kopidakis, N. \& Rumbles, G. Revealing the dynamics of charge carriers in polymer:fullerene blends using photoinduced time-resolved microwave conductivity. J. Phys. Chem. C 117, 24085-24103 (2013).
30. Dicker, G., de Haas, M. P., Siebbeles, L. D. A. \& Warman, J. M. Electrodeless time-resolved microwave conductivity study of charge-carrier photogeneration in regioregular poly(3-hexylthiophene) thin films. Phys. Rev. B 70, 045203 (2004).

31. Kroeze, J. E., Savenije, T. J. \& Warman, J. M. Electrodeless determination of the trap density, decay kinetics, and charge separation efficiency of dye-sensitized nanocrystalline TiO2. J. Am. Chem. Soc. 126, 7608-7618 (2004).

32. Yamamoto, Y. et al. Photoconductive coaxial nanotubes of molecularly connected electron donor and acceptor layers. Science 314, 1761-1764 (2006).

33. Ferguson, A. J., Kopidakis, N., Shaheen, S. E. \& Rumbles, G. Quenching of excitons by holes in poly(3-hexylthiophene) films. J. Phys. Chem. C 112, 9865-9871 (2008).

34. Holt, J. M. et al. Prolonging charge separation in P3HT-SWNT composites using highly enriched semiconducting nanotubes. Nano Lett. 10, 4627-4633 (2010).

35. Reid, O. G. \& Rumbles, G. Quantitative transient absorption measurements of polaron yield and absorption coefficient in neat conjugated polymers. J. Phys. Chem. Lett. 4, 2348-2355 (2013).

36. Treat, N. D. et al. Microstructure formation in molecular and polymer semiconductors assisted by nucleation agents. Nat. Mater. 12, 628-633 (2013).

37. Reid, O. G., Pensack, R. D., Song, Y., Scholes, G. D. \& Rumbles, G. Charge photogeneration in neat conjugated polymers. Chem. Mater. 26, 561-575 (2014).

38. Oga, H., Saeki, A., Ogomi, Y., Hayase, S. \& Seki, S. Improved understanding of the electronic and energetic landscapes of perovskite solar cells: high local charge carrier mobility, reduced recombination, and extremely shallow traps. J. Am. Chem. Soc. 136, 13818-13825 (2014).

39. de Haas, M. P. \& Warman, J. M. Photon-induced molecular charge separation studied by nanosecond time-resolved microwave conductivity. Chem. Phys. 73 35-53 (1982).

40. Schins, J. M. \& Talgorn, E. Conductive response of a photo-excited sample in a radio-frequent driven resonance cavity. Rev. Sci. Instrum. 82, 064703 (2011).

41. Saeki, A., Yasutani, Y., Oga, H. \& Seki, S. Frequency-modulated gigahertz complex conductivity of $\mathrm{TiO} 2$ nanoparticles: interplay of free and shallowly trapped electrons. J. Phys. Chem. C 118, 22561-22572 (2014).

42. Ferguson, A. J. et al. Trap-limited carrier recombination in single-walled carbon nanotube heterojunctions with fullerene acceptor layers. Phys. Rev. B 91, 245311 (2015).

43. Manzoni, C. et al. Intersubband exciton relaxation dynamics in single-walled carbon nanotubes. Phys. Rev. Lett. 94, 207401 (2005).

44. Scholes, G. D. Insights into excitons confined to nanoscale systems: electronhole interaction, binding energy, and photodissociation. ACS Nano 2, 523-537 (2008).

45. Park, J., Deria, P. \& Therien, M. J. Dynamics and transient absorption spectra signatures of the single-wall carbon nanotube electronically excited triplet state. J. Am. Chem. Soc. 133, 17156-17159 (2011).

46. Stich, D. et al. Triplet-triplet exciton dynamics in single-walled carbon nanotubes. Nat. Photon. 8, 139-144 (2014).

47. Izard, N. et al. Semiconductor-enriched single wall carbon nanotube networks applied to field effect transistors. Appl. Phys. Lett. 92, 243112 (2008).

48. Zhou, X., Park, J.-Y., Huang, S., Liu, J. \& McEuen, P. L. Band structure, phonon scattering, and the performance limit of single-walled carbon nanotube transistors. Phys. Rev. Lett. 95, 146805 (2005)

49. Ferguson, A. J. et al. Photoinduced energy and charge transfer in P3HT:SWNT composites. J. Phys. Chem. Lett. 1, 2406-2411 (2010)

50. Kroeze, J. E., Savenije, T. J., Vermeulen, M. J. W. \& Warman, J. M. Contactless determination of the photoconductivity action spectrum, exciton diffusion length, and charge separation efficiency in polythiophene-sensitized $\mathrm{TiO} 2$ bilayers. J. Phys. Chem. B 107, 7696-7705 (2003).

51. Piris, J. et al. The locus of free charge-carrier generation in solution-cast $\mathrm{Zn1-xMgxO/poly(3-hexylthiophene)} \mathrm{bilayers} \mathrm{for} \mathrm{photovoltaic} \mathrm{applications.}$ Adv. Funct. Mater. 17, 3849-3857 (2007).

\section{Acknowledgements}

This work was supported by the Solar Photochemistry Program of the U.S. Department of Energy, Office of Science, Basic Energy Sciences, Division of Chemical Sciences, Geosciences and Biosciences, under Contract Number DE-AC36-08GO28308 to NREL

\section{Author contributions}

J.P., J.L.B. and G.R. conceived the experiments. J.P. designed and performed the experiments, and analysed data. O.G.R. simulated solution-phase $f p$-TRMC cavity response. All authors wrote the manuscript.

\section{Additional information}

Supplementary Information accompanies this paper at http://www.nature.com/ naturecommunications 
Competing financial interests: The authors declare no competing financial interests.

Reprints and permission information is available online at http://npg.nature.com/ reprintsandpermissions/

How to cite this article: Park, J. et al. Photoinduced spontaneous free-carrier generation in semiconducting single-walled carbon nanotubes. Nat. Commun. 6:8809 doi: 10.1038/ncomms9809 (2015). (c) (i) This work is licensed under a Creative Commons Attribution 4.0 International License. The images or other third party material in this article are included in the article's Creative Commons license, unless indicated otherwise in the credit line; if the material is not included under the Creative Commons license, users will need to obtain permission from the license holder to reproduce the material. To view a copy of this license, visit http://creativecommons.org/ licenses/by/4.0/ 